3 Research Square

\title{
Clinical Outcome and
}

\section{Histopathologic Evaluations of Porous Tantalum Implant for the Treatment of Early-Stage Osteonecrosis}

\section{Yu Zhang}

Xuzhou Medical College Affiliated Hospital

Jian-Ning Sun

Xuzhou Medical College Affiliated Hospital

\section{Ye Zhang}

Xuzhou Medical College Affiliated Hospital

\section{Zi-Jian Hua}

Hebei Medical University Third Affiliated Hospital

Shuo Feng

Xuzhou Medical College Affiliated Hospital

Xiang-yang Chen ( $\nabla$ xzchenxiangyang@163.com )

Affiliated Hospital of Xuzhou Medical University https://orcid.org/0000-0001-7376-6179

\section{Research article}

Keywords: Osteonecrosis of the femoral head, Porous tantalum rod implantation, survival analysis, Total hip replacement

Posted Date: February 28th, 2020

DOI: https://doi.org/10.21203/rs.3.rs-15463/v1

License: (c) (i) This work is licensed under a Creative Commons Attribution 4.0 International License. Read Full License 


\section{Abstract}

Background To evaluate the survival rate of porous tantalum rod implantation in the treatment of avascular necrosis of the femoral head, to evaluate its clinical effect and imaging results, and to analyze the reasons for its failure to return to total hip arthroplasty (THA).

Methods From January 2008 to December 2013, tantalum rod implantation for avascular necrosis of the femoral head was performed in two institutions. Statistical analysis of operation data, including operation time, blood loss and blood transfusion. Harris hip score and imaging results were evaluated. Kaplan Meier survival analysis was performed with THA as the end point. Histopathological observations were performed on femoral head specimens with the tantalum rods that failed THA after tantalum rod implantation.

Results 42 patients (52 hips) were followed up completely, the average follow-up time was 74.3 months (60-120 months). 24 hips turned to THA at the end of follow-up (46.2\%), the average time was (44 \pm 32 ) months, and the average Harris score before THA was $(57 \pm 10)$. The follow-up time of 28 patients without THA was $78.6 \pm 13.8$ months, and the average Harris score was $80.6 \pm 2.8$. Histopathological examination revealed the implant surface is in contact with sparse Island bone. There is limited bone tissue extending inward from the implant. In the living bone area, there was "on-growth" of new bone but no "in-growth". In the necrotic area, there was no obvious new bone regeneration.

Conclusion The mid-and-long term clinical effect of tantalum rod implantation in the treatment of avascular necrosis of the femoral head is not good, and the osteogenic activity of tantalum rod in the femoral head is limited. ARCO stage, age and bone marrow edema were risk factors for the failure of tantalum rod implantation to THA.

\section{Introduction / Materials And Methods}

Osteonecrosis of the femoral head (ONFH) is a kind of pathological state with multiple causes, which leads to the decrease of blood supply of the subchondral bone of the femoral head, the death of bone cells and the collapse of the articular surface ${ }^{1,2}$. For the early and middle stage of non-invasive avascular necrosis of the femoral head, the first choice is to retain the femoral head. For the treatment of early avascular necrosis of the femoral head, the main principle is to effectively improve the blood supply of the femoral head and the function of the hip joint, and delay the development of the pathological process of necrosis of the femoral head. At present, several important factors for the treatment of avascular necrosis of the femoral head are as follows: $\otimes$ increase of new bone regeneration and neovascularization. $\otimes$ Remove the dead bone thoroughly. $\otimes$ Reduce the internal pressure of femoral head and promote the internal venous return of hip joint. $\otimes$ Maintain and increase the surface supporting force of the femoral head, prevent or treat the collapse of the femoral head $\mathrm{d}^{3,4}$.

There are many methods for the early necrosis of the femoral head, including hyperbaric oxygen, shock wave and other conservative treatment ${ }^{5-7}$. Surgical operations include osteotomy, vascularized bone flap 
implantation, and core decompression ${ }^{7-10}$. At present, it is generally recognized that core decompression can reduce the pressure in the bone, remove the necrotic bone tissue, increase the growth of blood vessels in the femoral head, and promote the crawling replacement of new tissue, which can be rapidly pain relieved, but the clinical satisfaction rate of using core decompression alone is not excited. The main reason is that although simple decompression can relieve the pressure in the femoral head, it can reduce the internal supporting force of the femoral head. Therefore, bone grafting or artificial materials are needed to provide enough biological support for the joint surface of the femoral head. According to Hungerford et al. ${ }^{11}$, core decompression combined bone grafting are suitable for the treatment of early avascular necrosis of the femoral head, which can effectively prevent the collapse of the femoral head and delay the time of hip replacement in the later stage. However, core decompression combined bone grafting often fail to provide effective bone support for the femoral head of patients. If the necrosis area is too large, the autogenous cancellous bone particles implanted after curettage of necrotic bone cannot provide effective subchondral support for the patients with core decompression combined bone grafting, and the autogenous iliac bone absorption implanted after operation is also the main reason for the collapse and deformation of the femoral head ${ }^{12}$.

Tantalum is a blunt metal with good biocompatibility. Bone and vascular tissue can be seen to grow rapidly in the hip joint with tantalum coating ${ }^{13,14}$. Core decompression combined with tantalum rod placement can release the internal pressure of the femoral head through core decompression, so as to alleviate the pain. Tantalum metal has the same elastic modulus as bone, and has the structure of bone trabecula, with high porosity. The tantalum rod designed by Zimmer company in the United States has a cylindrical structure with an aperture of $430 \mu \mathrm{m}$ and a diameter of $10 \mathrm{~mm}$, with a porosity of $75 \%-80 \%$, and a length of $70-130 \mathrm{~mm}$ ( $5 \mathrm{~mm}$ increase). The elastic modulus is equivalent to that of human fibula (3GPa), which has a good support for the femoral head ${ }^{15}$. Tantalum rod can bear physiological load of human body, has good biocompatibility and good friction stability. The placement of tantalum rod can play a role in filling the bone at the core and supporting the femoral head, reducing the stress distribution of the surrounding bone tissue, and effectively preventing the surface of the femoral head from collapsing. At the same time, the porous structure of tantalum rod can induce osteoblasts to grow in, accelerate the regeneration of blood vessels and promote the process of vascularization, which is conducive to the regeneration of femoral head and the repair of femoral head.

Many studies have reported that tantalum rod implantation has achieved good early clinical effect in the treatment of avascular necrosis of the femoral head $4,16,17$. However, the long-term clinical effect, weightbearing time and effect of porous tantalum implant are still controversial ${ }^{18-20}$. In the past few years, the failure rate of tantalum rods in ONFH has been reported to vary from $2 \%$ to $56 \%^{16,21}$. Once the subchondral bone collapses, the progression of the disease is difficult to reverse. The collapse and deformation of the femoral head, the narrowing of the joint space, and the deterioration of the joint function occur in turn. Hip replacement has become the only treatment option for these patients.

Moreover, at present, only animal studies have confirmed the bone ingrowth of porous tantalum rods ${ }^{13}$, 
proving that porous tantalum rods implantation is related to rapid and extensive bone growth, but the bone ingrowth in human body is still unclear.

In this study, the survival data of patients with avascular necrosis of the femoral head treated by tantalum rod implantation were reviewed and analyzed, and the clinical and imaging results were evaluated. The effect of tantalum rod implantation on avascular necrosis of the femoral head and the related factors leading to its conversion to THA were analyzed. In addition, pathological sections of the femoral head with tantalum rod were evaluated to confirm the degree of bone growth of tantalum rod in human body, and to evaluate the mid-and-long term efficacy of porous tantalum rod in the treatment of early avascular necrosis of the femoral head.

\subsection{Patient Selection}

From January 2008 to December 2013, patients with avascular necrosis of the femoral head underwent tantalum rod implantation in two hospitals were analyzed retrospectively. Inclusion criteria: non traumatic avascular necrosis of the femoral head, International Association of bone circulation stage (ARCO) I II patients. Exclusion criteria: skin lesions in the surgical area, active infections, coagulation disorders, and cases previously treated with any other type of treatment. This retrospective study was approved by the ethics committee of our institute, and we confirm that all methods are carried out in accordance with relevant guidelines and regulations. All patients signed the informed consent of operation.

One patient (2 hips) died of diseases unrelated to the operation, and three patients (3 hips) lost their visit. A total of 42 cases (52 hips; 5 females and 37 males) were analyzed. The average age of these patients was 40.3 years (range: 20-63 years). There were 30 males and 12 females with an average body mass index of $(25 \pm 3) \mathrm{kg} / \mathrm{m}^{2}$. The etiology of osteonecrosis was idiopathic or unknown in 10 hips, the use of corticosteroids in 19 hips and alcohol abuse in 23 hips. The demographic data and preoperative baseline characteristics of patients are shown in Table 1.

\subsection{Surgical methods}

After anesthesia, the patient was in supine position, the hip was sterilized and covered with towel, and the skin protection film was applied. A $4 \mathrm{~cm}$ long incision was made $2 \mathrm{~cm}$ below the trochanter to separate the fascial muscle layer by layer from the proximal and lateral cortex of the femur. The intersections of the horizontal line slightly above the trochanter and the lateral femoral cortex were used as the insertion points, and the center of the osteonecrosis area was used as the orientation of the insertion points. In order to eliminate the influence of the anteversion angle of the femoral neck on the position, the hip should be rotated $10^{\circ} \sim 15^{\circ}$, and the guide pin should be inserted in the central coronal plane of the femoral neck; the $3.2 \mathrm{~mm}$ guide pin should be inserted $5 \mathrm{~mm}$ below the articular cartilage of the femoral head. Ream the hole along the direction of the guide pin, and use the hollow drill to gradually ream the marrow from $8 \mathrm{~mm}$ diameter to $9 \mathrm{~mm}$; in the process of reaming, clean the hollow drill to prevent bone fragments from gathering in it. When the hollow drill enters the necrotic bone and does not penetrate the necrotic area, the necrotic tissue is removed through the bone tunnel with a multi-directional curette. 
Reaming with $10 \mathrm{~mm}$ hollow drill. Insert a depth gauge to determine the length of the required tantalum rod, and the measured length shall avoid the tail of the tantalum rod finally exposed outside the lateral cortex, so as to avoid the pain caused by its abrasion of surrounding soft tissue. Install the corresponding length of the tapping extension at the front end of the tapping, and rotate clockwise to make all the threads enter the femur. Screw in the tantalum rod (product of Zimmer company of the United States) so that its tail is slightly niche into the bone cortex; if there is resistance when transplanting, determine whether the tapping is in place or whether there is broken bone in the medullary canal. Fluoroscopy showed that the tantalum bar was in good position, and the incision was sutured layer by layer. The second generation cephalosporin antibiotics were given to prevent incision infection 30 minutes before operation and 24 hours after operation. In 12 weeks after operation, the patients' hip joint was fully limited in weight-bearing, and functional exercise was performed without weight-bearing. After 12 weeks, they walked with full weight-bearing.

The indications of THA were: continuous hip pain interfered with daily activities and deterioration of hip score, or radiation collapse of femoral head and intraarticular penetration of tantalum rod. The technology of tantalum rod transfer to THA after failure includes femoral neck osteotomy and implant cutting, both of which use a power saw to remove the tantalum rod from the rotor with a special ring drill. This procedure is performed as a routine hip replacement.

\subsection{Clinical Assessment}

The follow-up examination was arranged at 1 month, 3 months, 6 months and 12 months, and then every 6 months. The evaluation parameters included Harris hip score and imaging and MR examination of the diseased hip. The X-ray films of hip joint were used to evaluate the size of lesions, the consistency of femoral head, whether there was crescent sign and the degeneration of hip joint. MR images were used to assess changes in bone marrow edema and lesion size. According to the ARCO ${ }^{23}$ grading system, the initial stage and the degree of involvement of the femoral head were evaluated by radiology. More than $30 \%$ of necrosis is defined as large osteonecrosis. Myeloedema was defined as a low signal area on T1 weighted images, The high signal region is defined on T2-weighted image or inversion recovery image区in the femoral head, neck and intertrochanteric region ${ }^{24,25}$. The clinical evaluation was conducted by a non surgeon observer throughout the study. Two radiologists who were not involved in the operation and did not know all the clinical information were measured radiographically. Each parameter is measured twice at appropriate intervals to prevent bias from affecting the results.

\subsection{Analysis of bone implant specimens}

The pathological section of the femoral head implanted with tantalum rod was completed in Shandong Key Laboratory of oral tissue regeneration by using the EXAKT hard tissue section system. The bone graft into the femoral head was fixed in $10 \%$ buffered formalin and prepared for histological analysis of thin sections without decalcification ${ }^{26}$. The section was mounted on a glass slide and gradually thinned by petrological grinding technology, stained by HE and Masson respectively, and examined by transmission 
optical microscope. This allows qualitative assessment of tissue response to tantalum implants, including calcification and fibrous tissue recognition, new bone formation, and vascular distribution and cellular structure of the femoral head.

\subsection{Statistical Analysis}

The data and charts were analyzed and processed by IBMS PSS Statistical 19.0 statistical software. Continuous variables were analyzed using independent sample $T$ test. Categorical variables were analyzed using the Pearson chi-square or Fisher exact tests. Kaplan-Meier survivorship analyses were used with the endpoint defined as reoperation with THA. Test level was set at both sides $a=0.05, P<0.05$ was considered statistically significant.

\section{Result}

\subsection{Basic conditions of surgery.}

The operation was successfully completed in all cases. The mean time was $51 \pm 14$ minutes (38-69 minutes), intraoperative bleeding volume was $80 \pm 21 \mathrm{ml}(45-170 \mathrm{ml})$, and hospital stay was $8 \pm 3$ days (5-14 days).

\subsection{Clinical Results}

The average follow-up time was 74.3 (60-120) months, and the preoperative score of 52 cases of hip joint was $(74 \pm 13) \llbracket 6$ months after treatment, Harris score was $(80 \pm 13)$ on average, 5.89 points higher than that before treatment, the difference was significant $(P<0.05) .12$ months after treatment, the average Harris score was ( $84 \pm 11$ ), which was significantly different from that before treatment. 36 months after treatment, the average Harris score was $(82 \pm 11)$, and the average score was $(69 \pm 15)$ at the end of follow-up or before THA. (Figure.1) According to the stage of ARCO before operation, the average score at the end of follow-up of patients with ARCO I was higher than that of patients with ARCO II $(P<0.05)$; the average score at the end of follow-up of patients with age $\leq 40$ years was significantly higher than that of patients with age $>40$ years $(P>0.05)$; there was no significant difference in Harris score at the end of follow-up of different causes $(P>0.05)$.

\subsection{Radiographic Results}

At the last follow-up, 28 of 52 hip joints showed imaging progress (53.8\%). Among them, 5 of 22 hips in ARCO I stage progressed to stage III and 3 to stage II; 12 of 30 hips in ARCO I stage progressed to stage III and 8 to stage IV. The imaging progress rate of hip joint in ARCO I stage $(36.4 \% ; 8 / 22)$ was significantly lower than that in ARCO $\otimes$ stage $(66.7 \% ; 20 / 30)\left(x^{2}=4.690, P<0.05\right)$. The progress rate of patients with age $>40$ years was significantly higher than that of patients with age $\leq 40$ years $\left(x^{2}=4.748, P<0.05\right)$. Among the 28 hip joints with imaging progress, 24 (89.1\%) needed THA finally, while none of the 24 hip joints without imaging progress needed THA. 


\subsection{Survivorship and factor analysis of transition to THA}

From 42 patients with 52 ONFH, THA was necessary in 24 hips (19 patients). This implies a survival rate of $52.9 \%$ after implantation of an osteonecrosis intervention rod. (Figure. 2,3) The mean time of THA was (44 \pm 32 ) months. Patients who need THA: 13 males, 6 females; 14 unilateral, 5 bilateral; 10 hormonal hips, 11 alcoholic hips, 3 idiopathic (osteonecrosis of the femoral head with unknown clinical direct cause); 5 in ARCO I stage and 19 in ARCO II stage. Before operation, 21 hips with Harris $<80,3$ hips with Harris $\geq 80 ; 11$ hips with age $\leq 40,13$ hips with age $>40 ; 19$ hips with bone marrow edema. The average age was $(44.8 \pm 5.6)$ years old, the average body mass index was $(25.3 \pm 4.9) \mathrm{kg} / \mathrm{m} 2$, the average operation time was $(55.9 \pm 8.7) \mathrm{min}$, the average amount of bleeding was $(97.0 \pm 28.0) \mathrm{ml}$, the average Harris score before operation was $(70.3 \pm 6.2)$, and the average Harris score at the end of follow-up was (57.1 \pm 7.6$)$.

The proportion of patients with different ARCO stage classification to THA was different. There was a significant difference between the two groups $\left(\mathrm{x}^{2}=8.421, \mathrm{P}<0.05\right)$. The proportion of patients over 40 years old who needed THA was higher than those under 40 years old $\left(x^{2}=4.748, P<0.05\right)$. The proportion of hips with bone marrow edema to THA was higher than that without bone marrow edema $\left(x^{2}=8.421\right.$, $P<0.05)$. 44.2\% (13/30) of the male patients needed THA, and $55.6 \%(7 / 12)$ of the female patients needed THA. There was no significant difference between the sexes $\left(x^{2}=0.773, P>0.05\right)$. There was no significant difference in the conversion to THA due to different etiology (hormone, alcohol, idiopathic) $\left(x^{2}=1.397, P>0.05\right)$, unilateral or bilateral $\left(x^{2}=0.120, P>0.05\right)$, preoperative Harris score $\geq 80\left(x^{2}=0.285\right.$, $P>0.05)$.

\subsection{Postoperative complications}

One patient developed deep infection after tantalum rod implantation at 2 months postoperatively. The result of bacterial culture was Staphylococcus epidermidis infection. The patients were treated with onestage tantalum rod removal and antibiotic cement chain removal, and sensitive antibiotics were used after the operation, followed by two-stage THA. No postoperative complications such as femoral neck fracture and intertrochanteric fracture were found in other patients.

\subsection{Histopathological evaluation}

When observing the gross specimen of the femoral head cut from the hip replacement, it was found that: cartilage and subchondral bone were separated, subchondral fracture and articular surface collapse; there was no repair phenomenon in the necrotic area, there was no new bone formation and blood vessel growth in the necrotic area, the front end of the tantalum rod in the necrotic area was separated from the dead bone, the articular surface collapsed, cartilage peeled off, and the front end of the tantalum rod was exposed (Figure. 4).

Histological examination confirmed the growth of bone into the porous system (Figure. 5). The implant surface is in contact with sparse Island bone. There is limited bone tissue extending inward from the implant. Normal bone cell morphology can be observed in the rod hole of tantalum (Fig.5 f ), which is the 
same as that of the peripheral bone cell morphology of the porous tantalum rod (Fig. $5 \mathrm{a}, \mathrm{b}$ ). The bone trabecula of the regenerated bone was identified in the support area, contacting the peripheral structure of the porous tantalum rod (Fig. 5 c, e). In the living bone area, there was "on-growth" of new bone but no "ingrowth". In the necrotic area, there was no obvious new bone regeneration (Fig. $5 \mathrm{~d}$ ), and bone ingrowth only occurred in the implant part of femoral neck.

\section{Discussions}

Tantalum rod implantation, as a kind of hip joint preserving operation, has good biocompatibility, elastic modulus similar to fibula, subchondral bone support and core decompression effect, and has achieved good short-term effect in clinic ${ }^{27}$. However, the long-term effect of tantalum rod implantation is uncertain. Tanzer et al. ${ }^{26}$ conducted histopathological analysis on 15 cases of tantalum rod removed due to clinical failure, and found that there was no new bone formation and blood vessel growth in the femoral head with large area of necrosis; the diameter of tantalum rod was only $10 \mathrm{~mm}$, and its mechanical support range was limited, which could not provide enough subchondral bone support for the femoral head with large area of necrosis; in the absence of In the area supported by tantalum rods, the collapse of the femoral head will continue to progress. After that, it has been reported that TA rod implantation failed to transfer to THA in the treatment of femoral head necrosis 28,29 .

The prognosis of osteonecrosis of the femoral head is related to age, gender, etiology and preoperative stage $^{30}$. The results of this study show that the prognosis of tantalum rod implantation is related to the age of patients, bone marrow edema and ARCO stage before operation. The operative effect of hip joint preservation in osteonecrosis of femoral head is related to clinical stage. When the femoral head collapses before operation, the prognosis is poor ${ }^{31}$. Veillette et al. ${ }^{16}$ followed up 58 patients with tantalum rod implantation and found that in 49 Steinberg stage II, 6 (12\%) needed THA, and in 8 Steinberg stage III, 3 (38\%) needed THA. Patients in stage III were more likely to fail than those in stage II. Zhao et al. ${ }^{32}$ also pointed out that the survival rate of patients with ARCO IV stage (63.6\%) was significantly lower than that of patients with ARCO II stage $(95 \%)$ and ARCO III stage $(92 \%)(P<0.05)$. The results of this study showed that the survival rate of patients with ARCO stage II was lower than that of patients with stage $\mathrm{I}$, and the failure rate was higher, and the ARCO stage was a risk factor for conversion to THA. Florkemeier et al. ${ }^{33}$ followed up 19 patients ( 23 hips) who underwent tantalum rod implantation combined with core decompression for an average of 1.45 years. Although the patients included in this study were patients with early necrosis of the femoral head in ARCO I stage and II stage, the overall survival rate was only $44 \%$. It was pointed out that tantalum rod combined with core decompression did not show obvious advantages compared with core decompression alone. The advantages of early weight-bearing in this operation may be of clinical significance. As a result, they did not recommend tantalum rod implantation for osteonecrosis of the femoral head. A meta-analysis also concluded that the clinical effect of hip joint preservation surgery on patients with collapsed hip joint was poor ${ }^{34}$. 
Osteonecrosis of the femoral head is a progressive disease that easily affects young patients with age about 35 years ${ }^{35}$.The young patients have a good prognosis after hip conserving surgery. The follow-up results of Nadeau et al. ${ }^{28}$ showed that the average age of patients who failed to switch to THA for tantalum rod implantation was (50 \pm 12$)$ years old, and the average age of patients who did not fail was $(37 \pm 12)$ years old, with significant difference $(P<0.05)$. Age is one of the prognostic factors of tantalum rod implantation. Tsao et al. ${ }^{15}$ also pointed out that the older patients were prone to failure after tantalum rod implantation. Liu et al. ${ }^{29}$ followed up 44 patients ( 57 hips) with modified tantalum rod implantation for an average of 44.8 months, and failed 11 hips (19.3\%). The preoperative age did not affect the survival rate of the patients, and had nothing to do with the prognosis of the operation. The follow-up results of this study showed that patients with age $>40$ years old had higher failure rate and lower survival rate, and age was a risk factor for THA, suggesting that patients' age was one of the factors affecting the effect of tantalum rod implantation.

Previous studies have shown that bone marrow edema is a poor prognostic signal because it occurs after the onset or deterioration of hip pain and is associated with subsequent collapse of the femoral head, which may indicate progression to $\mathrm{ONFH}^{24,25,36}$. lida et al. ${ }^{37}$ reported that bone marrow edema did not exist in the initial MR imaging of osteonecrosis, and concluded that bone marrow edema should be considered as a sign of possible progression to advanced osteonecrosis. According to lto et al. ${ }^{25}$, the final imaging stage of 28 hip patients with bone marrow edema was significantly more advanced than that without bone marrow edema. Bone marrow edema is closely related to necrosis volume and is the most important risk factor for the aggravation of hip joint pain. Consistent with these studies, this study determined that bone marrow edema is the most important independent prognostic factor associated with THA demand. Although the number of bone marrow edema in the hip is limited in the present study, our results show that the survival rate of the hip with bone marrow edema is significantly lower than that without bone marrow edema.

By observing Tsao et al. 15 and our failure cases of tantalum rod hip, we can find that these cases have a common feature-large necrosis range. The diameter of tantalum rod is only $10 \mathrm{~mm}$, only occupying a small necrotic area (average $21 \%, 6 \%-58 \%$ ) 26 . When the necrotic area is large and there are trabecular fracture and subchondral bone microfracture in the femoral head, it is doubtful whether tantalum rod can provide sufficient support for subchondral bone plate and maintain the biomechanical stability in the femoral head. Aldegheri et al.38 think that tantalum rod is only suitable for young patients with Steinberg stage I and II whose articular surface is less than $30 \%$. When the necrosis area is small and the subchondral bone plate remains intact, tantalum rod can effectively provide mechanical support for the articular surface and start the repair process after necrosis. The authors also believe that preoperative underestimation of the necrotic range is the main cause of failure, because a single tantalum rod cannot support a wide necrotic range or Steinberg stage I and stage II multi focus necrotic lesions. Varitimidis et al. 4 also have similar findings. Their clinical research shows that the extent of necrosis is significantly related to the progress of postoperative imaging staging, and tantalum rod support does not block the progress of the disease 
Porous tantalum biomaterials have been proved to be related to rapid and extensive bone growth in several animal studies13, 22. In 1999, bobyn et al. 13 reported the rapid bone growth and fixation force after porous tantalum metal implants were implanted into animal models. The bone growth rate in the pores of dogs was $13 \%$ at 2 weeks, $42 \%-53 \%$ at 4 weeks, $63 \%-80 \%$ at 16 to 52 weeks, and the remodeling of Haversian canal could be seen at the later stage, and the shear fixation strength reached $18.5 \mathrm{mpa}$ at 4 weeks. These experimental data suggest that It shows that porous tantalum is very beneficial to the growth of bone and the combination with host bone. In 2008, wellDon et al. 14pointed out through cell culture that osteoblasts have a binding reaction with pure tantalum metal, which is a good substrate for the attachment, growth and differentiation of osteoblasts, and confirmed that tantalum is a long-term biocompatible metal, so it is also called bone trabecular metal. However, Tanzer et al. And ohkj et al. 26, 39showed that it was difficult to repair the necrosis area of the femoral head, especially the subchondral bone plate, the subchondral fracture and the collapse of the femoral head, and the length of the host bone into the tantalum rod was very limited. Similarly, the results of pathological section of tantalum rod of femoral head in this study are similar to this result tantalum rod in the femoral head is not good, and the osteogenic activity is limited. The repair of necrotic lesions is the ultimate goal of treatment and the key to the success of tantalum stick. Tantalum rod implantation of minimally invasive determines the intraoperative for the removal of the necrotic bone is limited, in order to improve the repair ability of necrosis area, how to remove dead bones in a limited decompression channel of the core and implant autologous, allogeneic or artificial bones containing drugs or biological factors that promote bone growth to enhance the repairing ability of necrotic bones, especially subchondral bone plates, is the future research direction, including the type of bone graft, the amount of bone graft, and whether to suppress it.

We acknowledge some limitations of this study. First, this is a nonrandomized retrospective study of patients treated by different surgeons in different institutions. Second, the number of subjects included in the study is too small, and further large-scale studies are needed to clarify the long-term clinical results of real-time porous tantalum implantation for $\mathrm{ONFH}$, as well as a large number of histological evidence indicating the bone ingrowth of porous tantalum implants. Finally, the study failed to establish a control group to evaluate whether porous tantalum implants are superior to other head saving surgery.

\section{Conclusion}

1. The clinical effect of tantalum rod implantation in the treatment of avascular necrosis of the femoral head is not good, the growth of tantalum rod in the femoral head is not good, and the osteogenic activity is limited.

2. ARCO stage, age and bone marrow edema were risk factors for the failure of tantalum rod implantation to THA.

\section{Abbreviations}

THA $\triangle$ Total hip arthroplasty $₫ \mathrm{ONFH}$ : Osteonecrosis of the femoral head 


\section{Declarations}

\section{Ethics approval and consent to participate}

This study was approved by the Ethics Committee of the Affiliated Hospital of Xuzhou Medical University and in accordance with the standards of the National Research Council. Written informed consent was obtained from all participants.

\section{Consent for publication}

Not applicable.

\section{Availability of data and materials}

We do not wish to share our data, because some of patient's data regarding individual privacy, and according to the policy of our hospital, the data could not be shared to others without permission.

\section{Competing interests}

The authors declare that they have no competing interests.

\section{Funding}

The author(s) received no financial support for the research, authorship, and/or publication of this article.

\section{Authors' contributions $\square$}

Yu Zhang did the study, analyzed the data, and wrote the manuscript. Ye Zhang, Jian-Ning Sun, Zi-Jian Hua, Shuo Feng, Xiang-Yang Chen were involved in the design, data management, and analysis of the study. Ye Zhang, Jian-Ning Sun, Zi-Jian Hua, Shuo Feng, Xiang-Yang Chen were involved in the study design, and data analysis. All authors read and approved the final manuscript.

\section{Acknowledgements}

We would like to acknowledge the helpful comments on this paper received from our reviewers.

\section{References}

1. Malizos KN, Karantanas AH, Varitimidis SE, Dailiana ZH, Bargiotas K, Maris T. Osteonecrosis of the femoral head: Etiology, imaging and treatment. European Journal of Radiology.63(1):0-28.

2. Cui L, Zhuang Q, Lin J, Jin J, Zhang K, Cao L, et al. Multicentric epidemiologic study on six thousand three hundred and ninety five cases of femoral head osteonecrosis in China. International Orthopaedics.40(2):267-76. 
3. Beckmann J, Goetz J, Baethis H, Kalteis T, Grifka J, Perlick L. Precision of computer-assisted core decompression drilling of the femoral head. Arch Orthop Trauma Surg.126(6):374-9.

4. Varitimidis SE, Dimitroulias AP, Karachalios TS, Dailiana ZH, Malizos KN. Outcome after tantalum rod implantation for treatment of femoral head osteonecrosis. Acta Orthopaedica.80(1):20-5.

5. Lai KA, Shen WJ, Yang CY, Shao CJ, Hsu JT, Lin RM. The use of alendronate to prevent early collapse of the femoral head in patients with nontraumatic osteonecrosis. A randomized clinical study. 2005;87(10):2155-9.

6. Reis ND, Schwartz O, Militianu D, Ramon Y, Levin D, Norman D, et al. Hyperbaric oxygen therapy as a treatment for stage-I avascular necrosis of the femoral head. 2003;86(1):371-5.

7. Wang, Ching-Jen. Treatment for Osteonecrosis of the Femoral Head: Comparison of Extracorporeal Shock Waves with Core Decompression and Bone-Grafting. Journal of Bone \& Joint Surgery American Volume.87(11):2380.

8. Korompilias AV, Lykissas MG, Beris AE, Urbaniak JR, Soucacos PN. Vascularised fibular graft in the management of femoral head osteonecrosis: TWENTY YEARS LATER. Journal of Bone \& Joint Surgery British Volume.91-B(3):287-93.

9. Zhao G, Yamamoto T, Ikemura S, Motomura G, Mawatari T, Nakashima Y, et al. Radiological outcome analysis of transtrochanteric curved varus osteotomy for osteonecrosis of the femoral head at a mean follow-up of 12.4 years. Journal of Bone and Joint Surgery - British Volume.92-B(6):781-6.

10. Dailiana ZH, Toth AP, Gunneson E, Berend KR, Urbaniak JR. Free Vascularized Fibular Grafting Following Failed Core Decompression for Femoral Head Osteonecrosis.22(5):0-688.

11. Hungerford DS. Response: The role of core decompression in the treatment of ischemic necrosis of the femoral head. Arthritis \& Rheumatology. 1989;32(6):801-6.

12. Hernigou P, Beaujean F, Lambotte JC. Decrease in the mesenchymal stem-cell pool in the proximal femur in corticosteroid-induced osteonecrosis. Journal of Bone \& Joint Surgery British Volume. 1999;81(2):349-55.

13. Bobyn JD, Stackpool GJ, Hacking SA, Tanzer M, Krygier JJ. Characteristics of bone ingrowth and interface mechanics of a new porous tantalum biomaterial. Journal of Bone \& Joint Surgery British Volume. 1999;81(5):907-14.

14. Welldon KJ, Atkins GJ, Howie DW, Findlay DM. Primary human osteoblasts grow into porous tantalum and maintain an osteoblastic phenotype. Journal of Biomedical Materials Research Part A. 2010;84A(3):691-701.

15. Tsao, A.K. Biomechanical and Clinical Evaluations of a Porous Tantalum Implant for the Treatment of Early-Stage Osteonecrosis. Journal of Bone \& Joint Surgery American Volume.87(suppl_2):22.

16. VEILLETTE CJH, MEHDIAN H, SCHEMITSCH EH, MCKEE MD. SURVIVORSHIP ANALYSIS AND RADIOGRAPHIC OUTCOME FOLLOWING TANTALUM ROD INSERTION FOR OSTEONECROSIS OF THE FEMORAL HEAD. The Journal of Bone and Joint Surgery. 2006;88:48-55.

17. Liu B, Sun W, Yue D, Li Z, Guo W. Combined Tantalum Implant with Bone Grafting for the Treatment of Osteonecrosis of the Femoral Head. Journal of Investigative Surgery.26(3):158-62. 
18. Liu Y, Yan L, Zhou S, Su X, Cao Y, Wang C, et al. Tantalum rod implantation for femoral head osteonecrosis: survivorship analysis and determination of prognostic factors for total hip arthroplasty. International Orthopaedics.40(7):1397-407.

19. Olsen M, Lewis PM, Morrison Z, McKee MD, Waddell JP, Schemitsch EH. Total hip arthroplasty following failure of core decompression and tantalum rod implantation. Bone Joint J.98-B(9):1175-9.

20. Zhang X, Wang J, Xiao J, Shi Z. Early failures of porous tantalum osteonecrosis implants: a case series with retrieval analysis. International Orthopaedics. 2016;40(9):1827-34.

21. Shuler MS, Rooks MD, Roberson JR. Porous Tantalum Implant in Early Osteonecrosis of the Hip: Preliminary Report on Operative, Survival, and Outcomes Results.22(1):0-31.

22. Bobyn JD, Toh K-K, Hacking SA, Tanzer M, Krygier JJ. Tissue response to porous tantalum acetabular cups - A canine model. Journal of Arthroplasty. 1999;14(3):347-54.

23. Mont MA, Hungerford DS. Non-traumatic avascular necrosis of the femoral head. The Journal of Bone and Joint Surgery. 1995;77(3):459-74.

24. Huang G, Chan W, Chang Y, Chang $C$, Yu J. MR imaging of bone marrow edema and joint effusion in patients with osteonecrosis of the femoral head: Relationship to pain. Ajr American Journal of Roentgenology. 2003;181(2):545-9.

25. Ito H, Matsuno T, Minami A. Relationship Between Bone Marrow Edema and Development of Symptoms in Patients With Osteonecrosis of the Femoral Head. Ajr American Journal of Roentgenology. 2006;186(6):1761-70.

26. Tanzer, Bobyn, JD, Krygier, JJ. Histopathologic Retrieval Analysis of Clinically Failed Porous Tantalum Osteonecrosis Implants. 2008;90(6):1282-9.

27. Bobyn JD, Poggie PA, Krygier JJ, Lewallen DG, Hanssen AD, Lewis RJ, et al. Clinical Validation of a Structural Porous Tantalum Biomaterial for Adult Reconstruction. 2004;86-A Suppl 2(suppl 2):123.

28. Nadeau M, Séguin C, Theodoropoulos JS, Harvey EJ. Short Term Clinical Outcome of a Porous Tantalum Implant for the Treatment of Advanced Osteonecrosis of the Femoral Head. 2007;10(1):410.

29. Liu Y, Su X, Zhou S, Wang L, Wang C, Liu S. A modified porous tantalum implant technique for osteonecrosis of the femoral head: survivorship analysis and prognostic factors for radiographic progression and conversion to total hip arthroplasty. 2015;8(2):1918-30.

30. Baksi DP. Treatment of osteonecrosis of the femoral head by drilling and muscle-pedicle bone grafting. Bone \& Joint Journal.73-B(2):241-5.

31. Tooke SMT, NUGENT PJ, BASSETT LW, NOTTINGHAM P, MIRRA J, JINNAH R. Results of Core Decompression for Femoral Head Osteonecrosis. Clin Orthop Relat Res.\&NA;(228):99???104.

32. Zhao D, Zhang Y, Wang W, Liu Y, Li Z, Wang B, et al. Tantalum Rod Implantation and Vascularized Iliac Grafting for Osteonecrosis of the Femoral Head. Orthopedics. 2013;36(6):789-95.

33. Floerkemeier $T$, Thorey F, Daentzer $D$, Lerch $M$, Klages $P$, Windhagen $H$, et al. Clinical and radiological outcome of the treatment of osteonecrosis of the femoral head using the osteonecrosis intervention 
implant.35(4):489-95.

34. Mont MA, Carbone JJ, Fairbank AC. Core Decompression Versus Nonoperative Management for Osteonecrosis of the Hip. Clin Orthop Relat Res.324(324):169-78.

35. Wilkins. Symptomatic Multifocal Osteonecrosis: A Multicenter Study. Clin Orthop Relat Res. 1999;369(369):312-26.

36. Koo KH, Ahn I-O, Kim R, Song H-R, Jeong S-T, Na J-B, et al. Bone Marrow Edema and Associated Pain in Early Stage Osteonecrosis of the Femoral Head: Prospective Study with Serial MR Images1. Radiology.213(3):715-22.

37. lida S, Harada Y, Shimizu K, Sakamoto M, Moriya H. Correlation Between Bone Marrow Edema and Collapse of the Femoral Head in Steroid-Induced Osteonecrosis. Ajr American Journal of Roentgenology. 2000;174(3):735-43.

38. Aldegheri R, Taglialavoro G, Berizzi A. The tantalum screw for treating femoral head necrosis: rationale and results.2(2-3):63-8.

39. Pandher DS, Oh K-J. A new mode of clinical failure of porous tantalum rod. Indian Journal of Orthopaedics.44(4):464.

\section{Table}

Table 1 Demographic and baseline characteristics 


\begin{tabular}{|c|c|}
\hline classification & Tantalum rod group \\
\hline Patients, $n$ & 42 \\
\hline Hips, n & 52 \\
\hline Age (years) & $40.3 \pm 10.3(20-63)$ \\
\hline \multicolumn{2}{|l|}{ Gender $\square \mathrm{n}(\%)$} \\
\hline Female & $12(11.9 \%)$ \\
\hline Male & $30(88.1 \%)$ \\
\hline BMI(ロ/口) & $25.4 \pm 2.6$ \\
\hline \multicolumn{2}{|l|}{ Age, $n(\%)$} \\
\hline$\square 40$ years & $36(69.2 \%)$ \\
\hline$\leq 40$ years & $16(30.8 \%)$ \\
\hline Bilateral disease, n (\%) & $32(61.54 \%)$ \\
\hline \multicolumn{2}{|l|}{ Etiology, $\mathrm{n}(\%)$} \\
\hline Corticosteroids & $19(36.54 \%)$ \\
\hline Alcoholism & $23(44.23 \%)$ \\
\hline Idiopathic & $10(19.23 \%)$ \\
\hline \multicolumn{2}{|l|}{ ARCO stage, n (\%) } \\
\hline$\square$ & $22(42.31 \%)$ \\
\hline 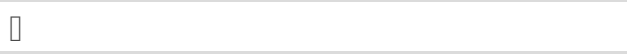 & $30(57.69 \%)$ \\
\hline Bone marrow edema & $30(57.69 \%)$ \\
\hline Osteonecrotic lesion size $\geq 30 \%, n$ (\%) & $21(40.38 \%)$ \\
\hline Harris hip score & $57.98 \pm 19.8$ \\
\hline \multicolumn{2}{|l|}{ Harris hip score, n (\%) } \\
\hline$\geq 80$ points & $8(15.38 \%)$ \\
\hline$<80$ points & $44(84.62 \%)$ \\
\hline Follow-up (months) & $74.3 \pm 4.5(60-120)$ \\
\hline
\end{tabular}

\section{Figures}




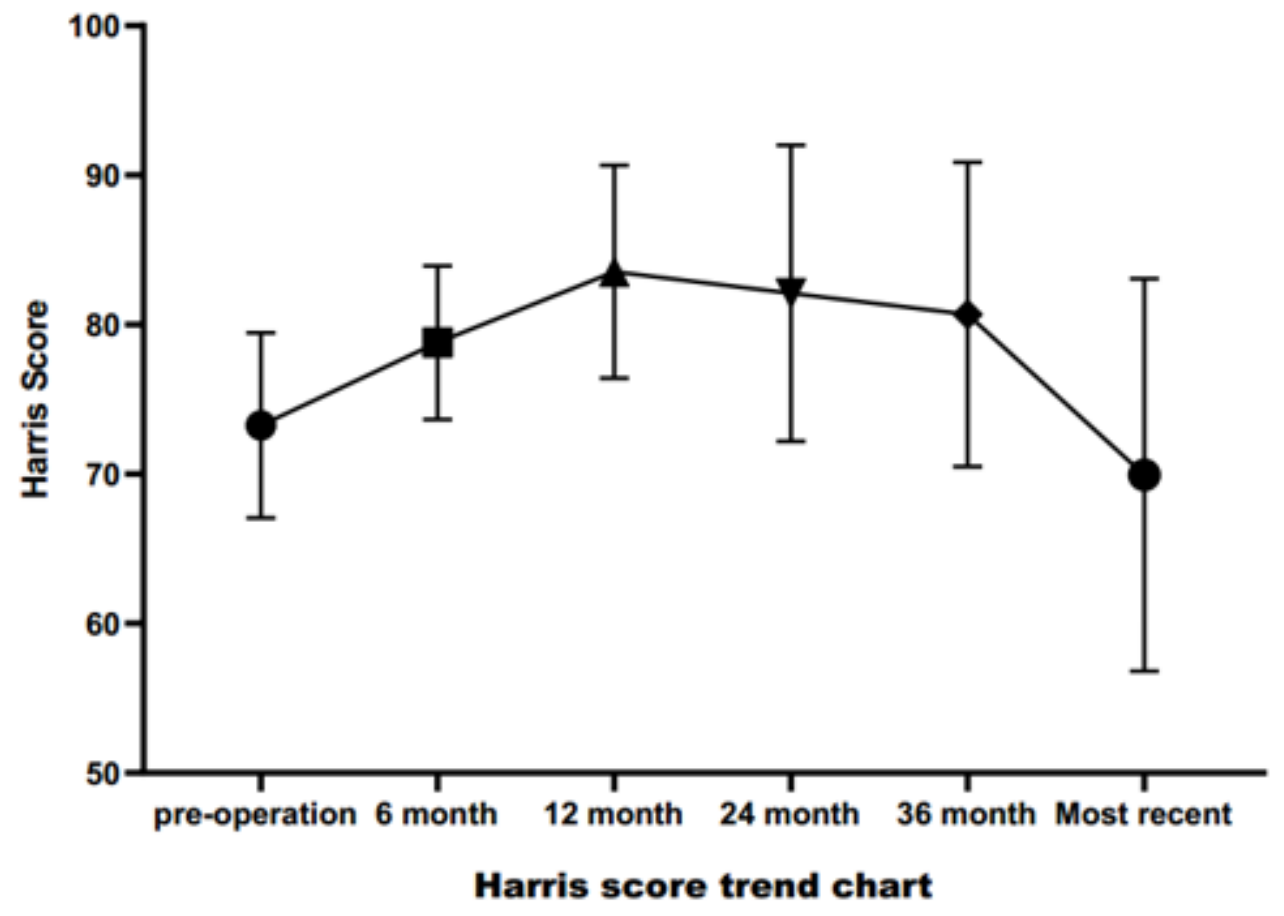

Figure 1

Trends of hip Harris score at different time points after operation

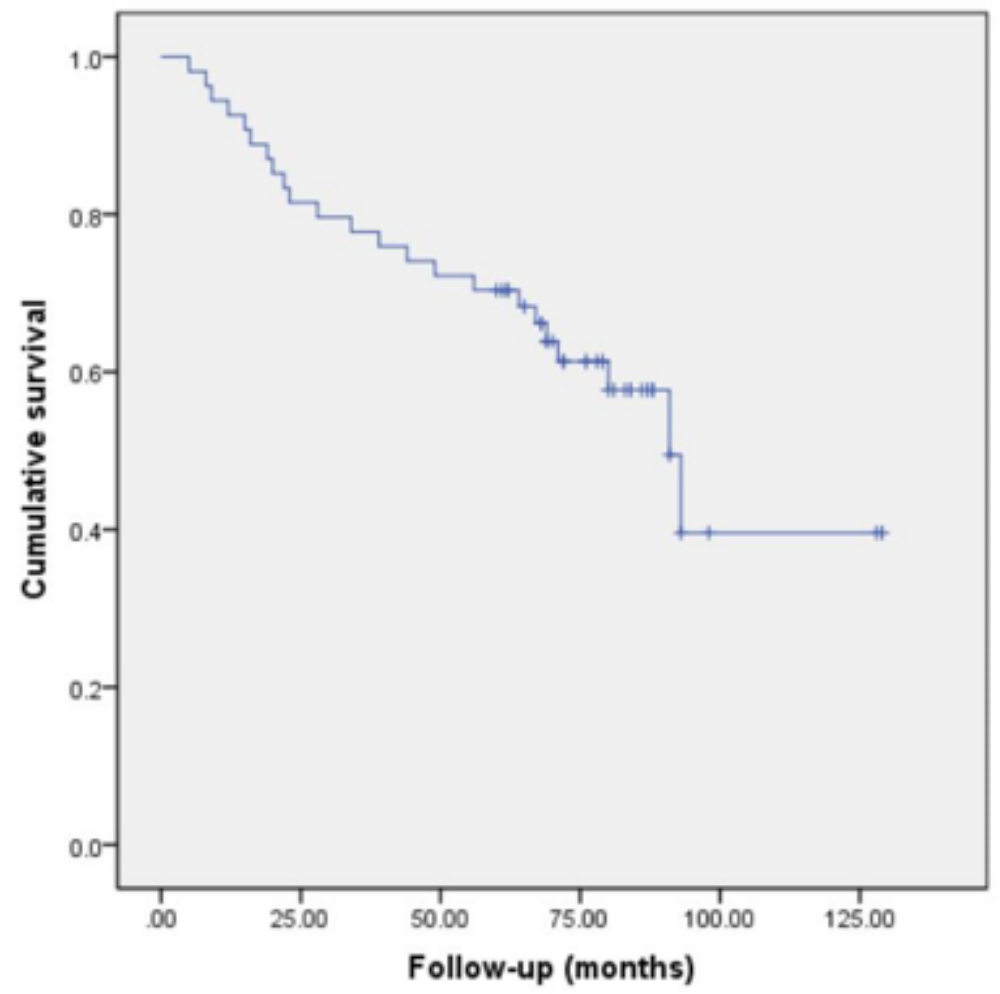

Figure 2

Kaplan-Meier survivorship curve with conversion to total hip replacement 


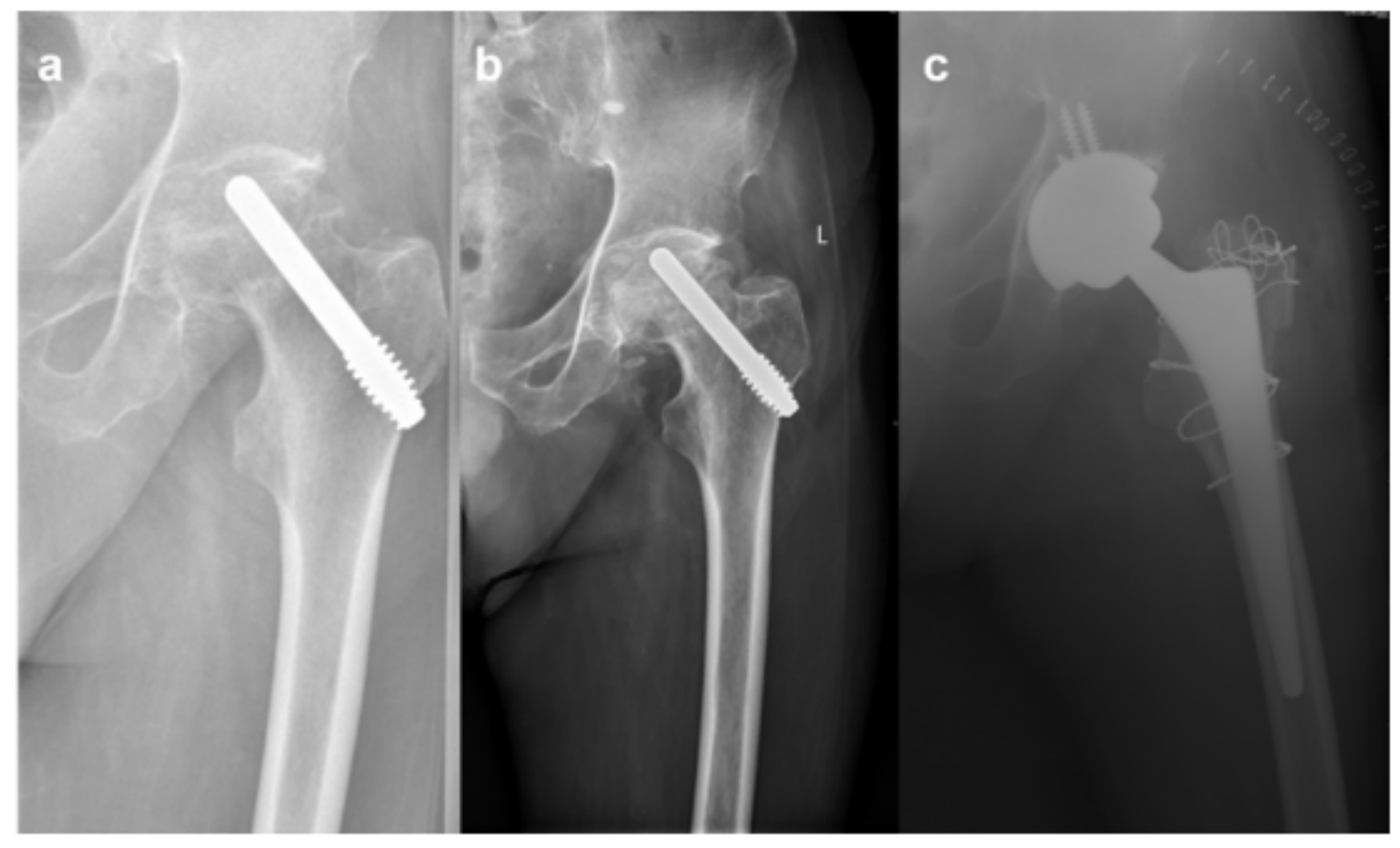

\section{Figure 3}

Follow-up of a patient with osteonecrosis of the femoral head (ONFH) after insertion of the tantalum implant with later conversion to total hip replacement

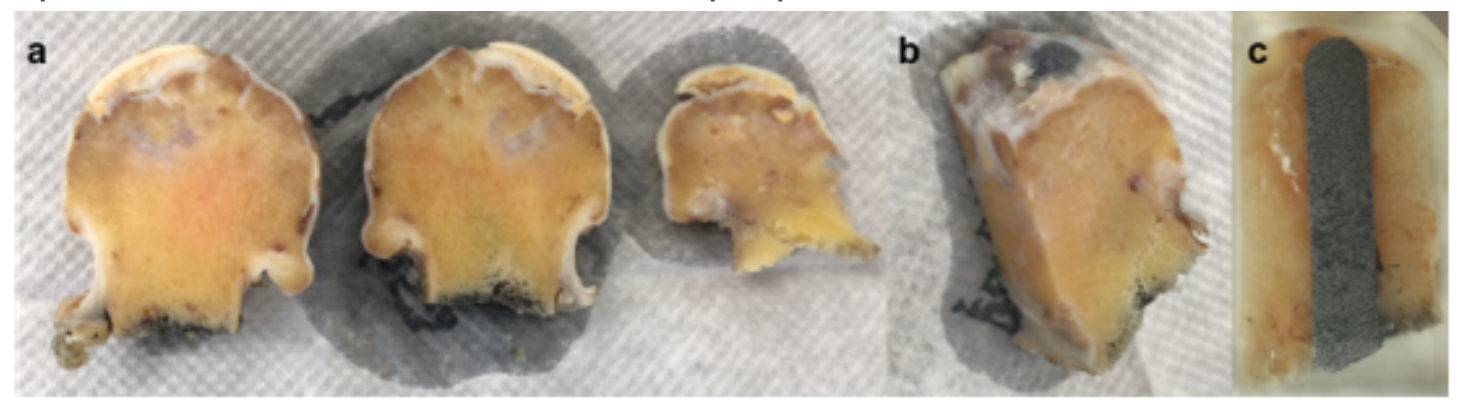

\section{Figure 4}

The gross specimen of the femoral head cut from the hip replacement with 129 months follow-up. a: Separation of cartilage and subchondral bone, subchondral fracture, articular surface collapse, no repair in necrotic area b: The cartilage is stripped off and the front end of the tantalum rod is exposed c: No obvious new bone in-grow was found in tantalum rod 


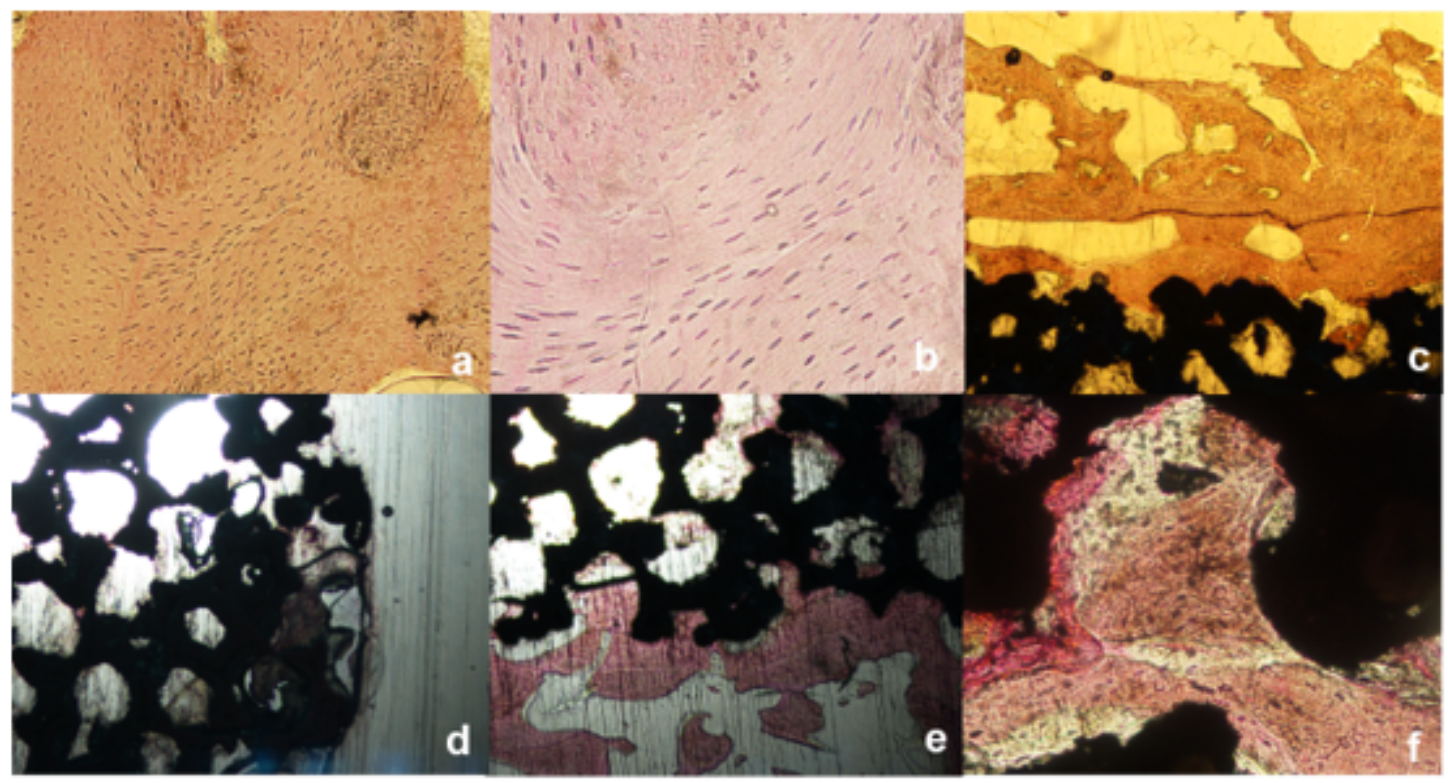

Figure 5

Histological examination囚retrieved after 129 months in situ. a, b: Peripheral normal bone cell morphology of tantalum rod c, e: The regenerated bone contacts the periphery of the porous tantalum rod d: No new bone regeneration was observed in the front end of tantalum rod f: Normal bone cell morphology was observed in the tantalum rod hole. 